ESJ Social Sciences

\title{
De la Degradation de l'habitat Social Comme Consequence de sa Politique d'entretien par les Syndics a Abidjan : Cas des Coproprietes Sogefiha d'Abobo et Sicogi d'Adjame
}

\author{
Adoua Affoua Krah Elisabeth
}

Doctorante en Sociologie Urbaine, Département de Sociologie Université Félix HOUPHOUËT-BOIGNY, Abidjan-Côte d'Ivoire

\section{Doi:10.19044/esj.2021.v17n23p177}

Submitted: 10 June 2021

Accepted: 01 July 2021

Published: 31 July 2021
Copyright 2021 Author(s)

Under Creative Commons BY-NC-ND

4.0 OPEN ACCESS

Cite As:

Adoua A.K.E. (2021). De la Degradation de l'habitat Social Comme Consequence de sa Politique d'entretien par les Syndics a Abidjan : Cas des Coproprietes Sogefiha d'Abobo et Sicogi d'Adjame. European Scientific Journal, ESJ, 17(23), 177.

https://doi.org/10.19044/esj.2021.v17n23p177

\section{Resume}

Entre 1960 et 1980, la Côte d'Ivoire met sur pied sa politique urbaine grâce à laquelle sont nées les copropriétés SICOGI (Société Ivoirienne de Construction et de Gestion Immobilière) et SOGEFIHA (Société de Gestion Financière de l'Habitat). A partir de 1986, ces 02 structures se retirent de la gestion des copropriétés, désormais confiée aux habitants eux-mêmes à travers l'installation des syndics. Mais aujourd'hui, l'on constate que ces espaces se trouvent dans un état de « dégradation » dû au manque d'entretien dont ils sont sujets. Cet article a pour objectif d'étudier la politique d'entretien des syndics à l'origine de la dégradation des espaces en copropriétés SOGEFIHA d'Abobo et SICOGI d'Adjamé 220 Logements. La recherche documentaire, l'observation directe ainsi que l'entretien ont été utilisés pour recueillir les données sans omettre la mobilisation de la gouvernance urbaine comme théorie de l'étude. Les résultats font ressortir que la dégradation est une conséquence des stratégies d'entretien ainsi que du manque de connaissance des syndics.

Mots clés : Dégradation, habitat social, entretien, copropriété, Abidjan 


\title{
Of the Degradation of Social Habitat as a Consequence of its Maintenance Policy by Trustees in Abidjan: Case of Sogefiha of Abobo and Sicogi of Adjame Condominiums
}

\author{
Adoua Affoua Krah Elisabeth \\ Doctorante en Sociologie Urbaine, Département de Sociologie \\ Université Félix HOUPHOUËT-BOIGNY, Abidjan-Côte d'Ivoire
}

\begin{abstract}
Between 1960 and 1980, Côte d'Ivoire set up its urban policy, in which the SICOGI (Ivorian construction and property management company) and SOGEFIHA (Society of financial management of habitat) condominiums were born. From 1986, these 02 structures withdrew from the management of condominiums, now entrusted to the inhabitants themselves through the installation of trustees. But today, we see that these spaces are in a state of "degradation" due to the lack of maintenance to which they are subject. The objective of this article is to study the maintenance policy of trustees at the origin of the degradation of the SOGEFIHA condominium spaces of Abobo and SICOGI 220 Housing units of Adjamé. Documentary research, direct observation as well as interview were used to collect the data without omitting the mobilization of urban governance as theory of the study. The results show that the deterioration is a consequence of maintenance as well as the lack of knowledge of the trustees.
\end{abstract}

Keywords: Degradation, social housing, maintenance, condominium, Abidjan

\section{Introduction}

La Côte d'Ivoire, à l'instar des autres pays Africains, entame son processus de développement dès son accession à l'indépendance. A cet effet, une politique urbaine est mise sur pied avec un double objectif : avoir des villes modernes et loger décemment les populations de classe moyenne, incapables de le faire au prix du marché (Kouamé, 1983).

Dans cette dynamique, deux sociétés immobilières sont créées : la Société de Gestion Financière de 1'Habitat (SOGEFIHA) et la Société Ivoirienne de Construction et de Gestion Immobilière (SICOGI), respectivement en 1963 et en 1964. Ces structures sont chargées de la réalisation des programmes de logements sociaux de la Cote d'Ivoire (Parentau \& Charbonneau, 1992). Inspiré de l'architecture moderne occidentale, l'habitat est construit sous forme de copropriété, constitué de 
plusieurs bâtiments comprenant des espaces privatifs et collectifs. (Haeringer, 1985 ; Simon, 2013).

Après avoir bâti plus de 65000 logements $^{1}$, la SOGEFIHA et la SICOGI en assurent l'entretien. Il s'agit de l'entretien des espaces communs et verts (ravalement des façades, tonte du gazon) ainsi que la réalisation de certains travaux tels que le curage des caniveaux.

A partir de 1973, la SOGEFIHA et la SICOGI connaissent des difficultés dues à des dysfonctionnements internes et à la détérioration de la conjoncture internationale liée à la première augmentation du prix du pétrole (Soumahoro, 1997). Cela a pour conséquence le retrait de ces deux grandes structures de la gestion des copropriétés à partir de 1986 (Krekpa et al, 2016).

Après leur retrait, l'entretien est confié aux copropriétaires qui s'organisent en syndicats avec pour mission la gestion de leur cadre de vie. Au niveau structurel, la régulation des espaces en copropriétés s'inscrit dans un cadre normatif à travers des lois et décrets ${ }^{2}$. Par ailleurs, l'on assiste à la création en 2014 de la Direction du logement et de la copropriété chargée d'encadrer les syndics dans leur tâche et de veiller à l'application des règles de vie en copropriété ${ }^{3}$. Aussi, des opérations de ravalement des façades de certains logements ont été entreprises par le gouvernement la même année dans le district d'Abidjan en appui à la législation en vigueur. En plus des actions des syndics, il existe au sein de ces espaces, des associations de jeunes et de femmes qui leur viennent en appui. Leur mission est de veiller aussi à l'entretien du cadre de vie à travers des opérations de nettoyage organisées périodiquement.

Malgré les actions des syndics et des associations, l'on assiste à une dégradation de ces copropriétés. En effet, les constats montrent que les murs des façades des bâtiments sont défraichis. L'on note également la présence d'odeurs nauséabondes dues aux débordements des fosses septiques avec l'obstruction des toilettes des logements. Les escaliers et les rampes sont

${ }^{1}$ La SOGEFIHA réalisa près de 29100 unités de logements urbains en Côte d'Ivoire dont 26269 à Abidjan et la SICOGI a pu produire près de 36000 logements (Parentau \& Charbonneau,1992, p 421).

${ }^{2}$ Le premier décret concernant la politique de l'habitat social fut pris en 1949 par l'Office des Habitations Economiques (OHE) pour toute l'Afrique Occidentale Française. Les objectifs de l'OHE étaient de construire des logements salubres à bon marché et orientés vers l'accession à la propriété individuelle. En 1998, un autre décret fut pris, modifiant celui de 1949. Le 22 mars 2013, le gouvernement ivoirien a adopté un décret portant réglementation du statut de la copropriété qui fut modifié et complété par le décret n²014-26 du 22 janvier 2014. Ces textes ont récemment été abrogés par la loi n²019-576 du 26 juin 2019, instituant le code de la construction et de l'habitat, dont le livre 2 détermine le mode d'habitation en copropriété.

${ }^{3}$ Voir décret $\mathrm{n}^{\circ}$ 2014-515 du 15 septembre 2014, portant organisation du Ministère de la Construction, du Logement de l'Assainissement et de l'Urbanisme. 
presqu'inexistants au sein des bâtiments. Enfin, les murs et les dalles des appartements sont fissurés avec des fuites d'eau.

Si la majorité des travaux dans le domaine de l'habitat en Afrique a pour la plupart été basée sur comment produire des logements afin de répondre aux besoins des populations sans cesse croissantes dans les milieux urbains (Konaté, 2011 ; Prévost, 1985 ; Soumahoro, 1997 ; Manou-Savina, 1989) ; la présente étude, met plutôt l'accent sur la dégradation du bâti déjà existant. En effet, plusieurs structures interagissent au sein de ces espaces dans le cadre de l'entretien, pourtant le phénomène de dégradation persiste. Dès lors, comment les modes de gestion des espaces en copropriété SOGEFIHA d'Abobo et SICOGI d'Adjamé sont-ils à l'origine de la dégradation?

\section{Methodologie}

\subsection{Site à l'étude}

L'étude a été réalisée dans le district d'Abidjan, plus précisément dans les communes d'Abobo et d'Adjamé pour plusieurs raisons. Les copropriétés SOGEFIHA d'Abobo ont été choisies comme champ d'étude pour des raisons de maitrise du terrain et de faisabilité.

Concernant les copropriétés SICOGI 220 logements d'Adjamé, l'histoire laisse entrevoir que le Grand bloc constitue la première acquisition de la SICOGI. Elle représente donc la plus ancienne des copropriétés de cette société d'où l'intérêt qu'elle suscite pour nous. Aussi, la commune d'Adjamé abrite-elle le siège de la SICOGI situé non loin des tours 220 logements. Ce choix s'est donc opéré en rapport avec la dimension symbolique liée à l'histoire de cette structure immobilière.

Ces différentes copropriétés présentent dans l'ensemble les mêmes caractéristiques que les autres copropriétés issues de la politique urbaine d'après les indépendances, en ce qui concerne les difficultés liées à leur entretien. Par conséquent, en plus d'être le symbole de l'habitat social en Côte d'Ivoire, elles constituent des échantillons représentatifs du phénomène à l'étude, à savoir la dégradation. La figure ci-dessous localise nos différentes zones d'étude. 
Figure 1 : Localisation des zones d'étude

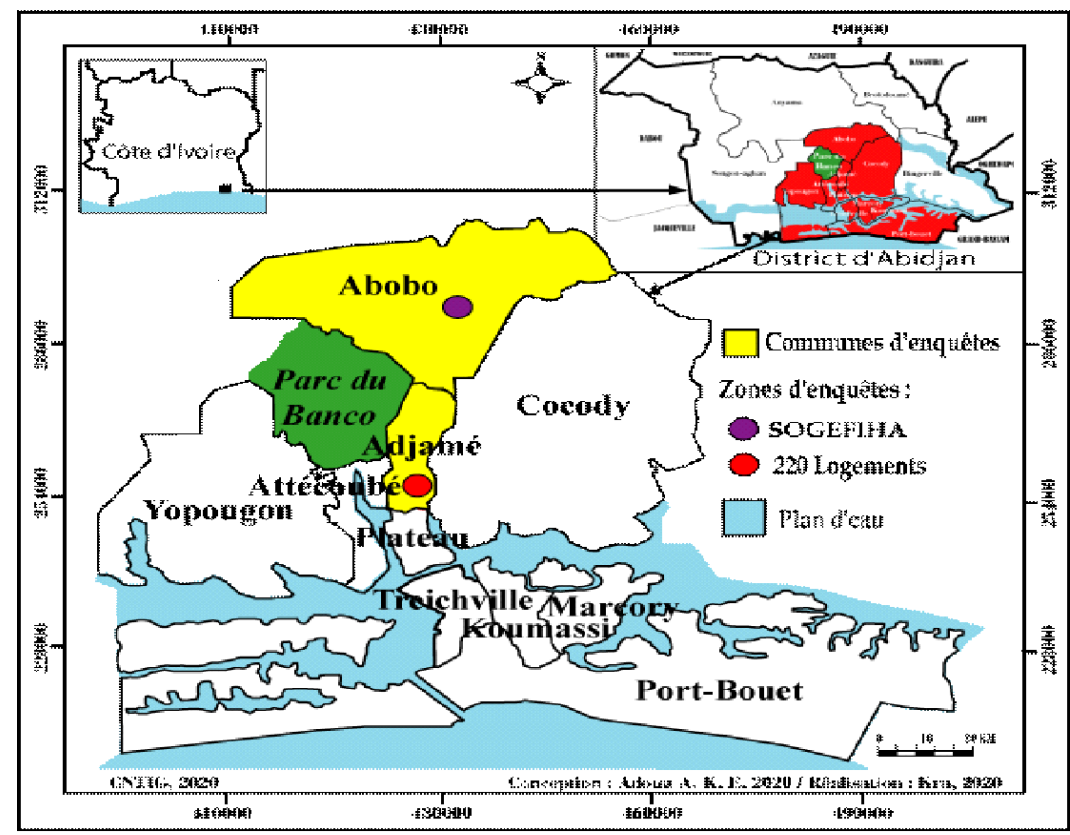

Conception : Adoua Elisabeth, septembre 2020

\subsection{Population à l'étude}

Dans cette étude, les habitants propriétaires, les habitants locataires, les présidents de syndics, les responsables d'associations et les acteurs institutionnels (agents de mairie, du ministère de la construction, du trésor et de la comptabilité publique, de la SICOGI, de la direction de la copropriété et de la SOGEPIE) ont été interrogés. A cet effet, on a fait ressortir les modes de gestions des copropriétés ainsi que les rapports entretenus entre les acteurs.

\subsection{Echantillonnage}

Cette étude s'inscrit dans une approche qualitative. De façon pratique, l'étude a été réalisée du 05 au 28 juillet 2019 auprès d'un échantillon de 52 personnes. La technique d'échantillonnage non probabiliste par choix raisonné a été mobilisé (N'da, 2015). De ce fait, le choix des habitants interrogés a été fait en fonction de leur ancienneté (durée), du rôle et du statut qu'elles occupent ainsi que des actions qu'elles ont menées à des périodes bien définies au sein desdites copropriétés. Les choix se sont donc portés sur les propriétaires, les héritiers, les locataires, les responsables de syndics et d'associations. Ainsi, 4 présidents de syndics, 6 responsables d'associations, 06 habitants-propriétaires et 24 locataires ont été interviewés. Cette technique repose sur le jugement du chercheur, qui fait le tri des cas à inclure dans l'échantillon, répondant de façon satisfaisante à sa recherche. Avec les acteurs 
institutionnels, il s'agissait de savoir le contexte historique de la construction des copropriétés ainsi que le mode de gestion de ces espaces avant de le désengagement de la SICOGI et la SOGEFIHA. A cet effet, 10 individus ont été interviewés.

\subsection{Posture théorique}

Ce travail s'inscrit dans le cadre de la gouvernance urbaine qui est un processus d'acteurs, de groupes sociaux et d'institutions en vue d'atteindre des objectifs définis et discutés collectivement (Le Gales 1995). Le choix de cette théorie s'est fait au regard de la multiplicité de groupes d'acteurs interagissant au sein des copropriétés étudiées dans le but de parvenir à leur gestion efficiente. Il s'agit des habitants de ces espaces (propriétaires, locataires), des bailleurs, des associations, des syndics et de l'acteur institutionnel. Il est question de montrer comment les modes de gestions et les rapports entre ces derniers constituent un mobile de la dégradation.

\subsection{Méthode d'analyse}

L'analyse de contenu thématique du discours (Bardin, 2007) des enquêtés a été privilégié dans ce travail. Les discours des enquêtés ont été analysés et interprétés dans leur contenu afin de comprendre le phénomène à l'étude. C'est-à-dire comment les modes de gestions des syndics induisent certaines pratiques qui ont progressivement conduit à la dégradation du cadre de vie. Aussi, la méthode structuro-fonctionnelle de Parsons (1951) a-t-elle été utilisée en vue de l'explication du phénomène à l'étude. Cette méthode a permis d'analyser le fonctionnement des différentes structures ainsi que des rapports qui en découlent dans la production de la dégradation des copropriétés SICOGI d'Adjamé et SOGEFIHA d'Abobo soumis à cette étude.

\subsection{Techniques et instruments de collecte de données}

L'étude se voulant qualitative, les techniques et instruments appropriés à ce cas de figure ont été utilisés. Ainsi, les techniques utilisées sont la recherche documentaire, l'observation directe et l'entretien. La recherche documentaire menée a contribué à une meilleure compréhension du sujet tout en permettant sa construction sociologique. L'observation a porté sur la structure spatiale des différentes copropriétés, l'état des espaces communs ainsi que les pratiques des habitants concernant l'entretien. Grâce à l'entretien (directif et semi-directif), les enquêtés ont pu produire des discours liés à leur vécu et à leur perception du phénomène à l'étude.

Les instruments mobilisés pour collecter les données de l'étude sont la grille de lecture, la grille d'observation et le guide d'entretien. La grille de lecture a permis de dresser la liste des idées retenues au cours des différentes lectures. Les comportements des habitants concernant l'entretien de leur cadre 
de vie ainsi que leurs pratiques sur les espaces communs ont été observés à partir de la grille d'observation. Le guide d'entretien a contribué à la compréhension et à l'analyse du phénomène grâce aux thématiques abordés.

\section{Resultats}

Les résultats de l'étude montrent que la dégradation est liée aux stratégies d'entretien mis sur pied par les syndics et les associations interagissant au sein des copropriétés SOGEFIHA d'Abobo et SICOGI 220 logements d'Adjamé ainsi qu'à leur manque de connaissance.

\subsection{Les stratégies d'entretien des copropriétés}

Les stratégies d'entretien mises en place par les syndics et les associations sont la mobilisation des fonds, de réunions relatives à la gestion du cadre de vie et d'activités d'entretien.

\subsubsection{La mobilisation des fonds}

Pour la mobilisation des fonds nécessaires à la gestion du cadre de vie, deux approches sont utilisées par les syndics des espaces étudiés.

La première réside en l'instauration de cotisation comme c'est le cas aux Quatre Etages d'Abobo. Au sein de cette copropriété, c'est l'association des femmes qui est chargée de recueillir l'argent auprès des habitants et la cotisation mensuelle est de 500 francs. Cependant, au fil du temps, les habitants ont arrêté de s'acquitter régulièrement de leurs cotisations et la somme recueillie à partir de la minorité qui cotise s'avère insuffisante pour couvrir les dépenses liées à l'entretien.

La seconde consiste à la mise en location des espaces communs. En effet, pour faire face aux charges d'entretien, les syndics des copropriétés d'Adjamé 220 Logements et d'Abobo N'Tanouan-Est mettent en location les espaces communs. Les espaces loués à Adjamé sont les espaces verts, la cour de la cité, trois (03) studios, vingt-deux (22) sous-sols, deux (02) magasins et l'espace non bâti utilisé par les maquis. C'est ce que nous confie le responsable du syndic en ces termes: "Le syndic vit des ressources générées par la location des sous-sols, des magasins, des studios et de l'espace utilisé pour les maquis ». (Homme, propriétaire, responsable du syndic à Adjamé Grand Bloc).

Au niveau de la copropriété N'Tanouan-Est d'Abobo, le syndic loue l'ancien espace vert transformé en terrain de sport comme lieu d'entrainements de trois clubs de football et pour la tenue de manifestations telles que : les veillées funèbres, les séances de prières et d'évangélisation, les concerts, etc...C'est ce qui ressort de ce discours : "Ce que nous faisons qui nous rapporte un peu de moyens c'est la location de l'espace qui sert de terrain. Il y'a des gens qui viennent faire des séances d'évangélisation, des 
veillées, .... C'est le fruit de ces locations qui nous permettent d'entretenir les caniveaux, c'est toujours par voie d'huissier que nous signifions à tous les copropriétaires qui sont en infraction de mettre fin aux constructions anarchiques. C'est cet argent que nous utilisons comme moyens pour le faire et pour mener les démarches administratives » (Homme, propriétaire, membre de syndic à Abobo SOGEFIHA N'Tanouan-Est). Toutefois, la somme recueillie n'aide qu'à couvrir une partie des charges d'entretien. La raison évoquée est que les locataires de ces espaces ne payent pas régulièrement leur dû. Ainsi, ces approches s'avèrent inefficaces car les fonds recueillis sont insuffisants et l'entretien demeure irrégulière et précaire. Cela a pour impact la dégradation progressive de ces espaces.

\subsubsection{Les réunions relatives à la gestion du cadre de vie}

Les réunions constituent des cadres d'échanges en vue de l'amélioration du cadre de vie. De ce fait, les réunions ordinaires des copropriétaires se tiennent une fois par trimestre et celles des associations sont mensuelles. Mais, les responsables sont confrontés à la faible participation des membres aux rencontres, ayant conduit dans certains cas à leur suspension.

A Abobo Quatre étages, sur 140 appartements, lorsque l'association en charge de l'entretien convoque une réunion, seules 20 personnes répondent. D'après la trésorière de l'association, «Quand on convoque une réunion, c'est une vingtaine qui vient et ce sont elles qui cotisent. Depuis qu'on a créé l'association il y'a des gens qui n'ont jamais cotisé » (Femme, propriétaire, présidente de l'AFUQUER ${ }^{4}$ ). Comme conséquence de la non-participation des habitants aux réunions et aux cotisations, la somme recueillie par mois s'avère insuffisante pour l'entretien. Ce désintérêt a créé un découragement chez les responsables de l'association au point où elles ont décidé de "rendre le tablier », c'est-à-dire de dissoudre l'association.

La même réalité est vécue à Adjamé, comme en témoigne ces dires : "Les réunions se faisaient dans les débuts de l'installation de notre bureau, mais ce sont les mêmes qui venaient donc on a arrêté. Il n'y a plus de réunion, $v u$ que nous sommes à peine une dizaine qui y prenons part» (Homme, propriétaire, responsable de syndic à Adjamé).

D'après ce verbatim, il n'existe plus de réunion au sein de cette copropriété à cause de la faible participation des habitants. Cette situation est problématique car c'est au cours de ces rencontres que les thèmes portant sur l'entretien sont discutés. Comme conséquence, l'entretien demeure l'affaire de quelques résidents uniquement et c'est l'état du cadre de vie qui se trouve dégradé. Ces différents discours laissent transparaitre que le nombre de participants aux rencontres a décru au fil du temps et sont révélateurs du

\footnotetext{
${ }^{4}$ AFUQUER : Association des Femmes Unis des Quatre Etages et Riveraines
} 
rapport des habitants à l'entretien de l'espace résidentiel. Leur désintérêt pour les questions liées à la gestion du cadre de vie a pour conséquence son délabrement.

\subsubsection{Les activités d'entretien}

Les activités d'entretien concernent le balayage de la cour, le ramassage des papiers et des plastiques ainsi que le curage des caniveaux. Ces activités sont organisées par les femmes regroupées en associations. A Abobo, nous avons l'Association des Femmes Unies des Quatre Etages et Riveraines (AFUQUER) et à Adjamé, l'Amicale des Femmes du Grand Bloc (AFGB). Dans l'ensemble, l'attitude des habitants constitue un frein à l'entretien et par la même occasion explique la dégradation et l'insalubrité de ces copropriétés. C'est ce que nous raconte cette habitante : "A la limite, les gens s'en foutent. Ils vont faire des travaux et ils jettent les débris dans la cour. Les gens continuent de balancer les poubelles par derrière, même les selles dans les sachets» (Femme, membre de l'AFUQUER). Une autre enquêtée nous instruit en ces termes : "Il y a un véritable problème de salubrité. Les jardins sont l'urinoir public. Nous tous, nous jetons tout, partout, l'eau ne monte plus chez mon voisin au dernier. Quand ils défèquent, ils jettent en bas ici» (Femme, responsable de l'AFGB). Ces dires montrent qu'en plus de ne pas participer à l'entretien, les habitants ne respectent pas les efforts fournis par ceux qui essaient de prendre soin du cadre de vie. Dès lors, les facteurs susmentionnés expliquent la dégradation des copropriétés étudiés comme l'illustre la photo 1 suivante.

Photo 1 : Débordement d'un regard à Abobo SOGEFIHA

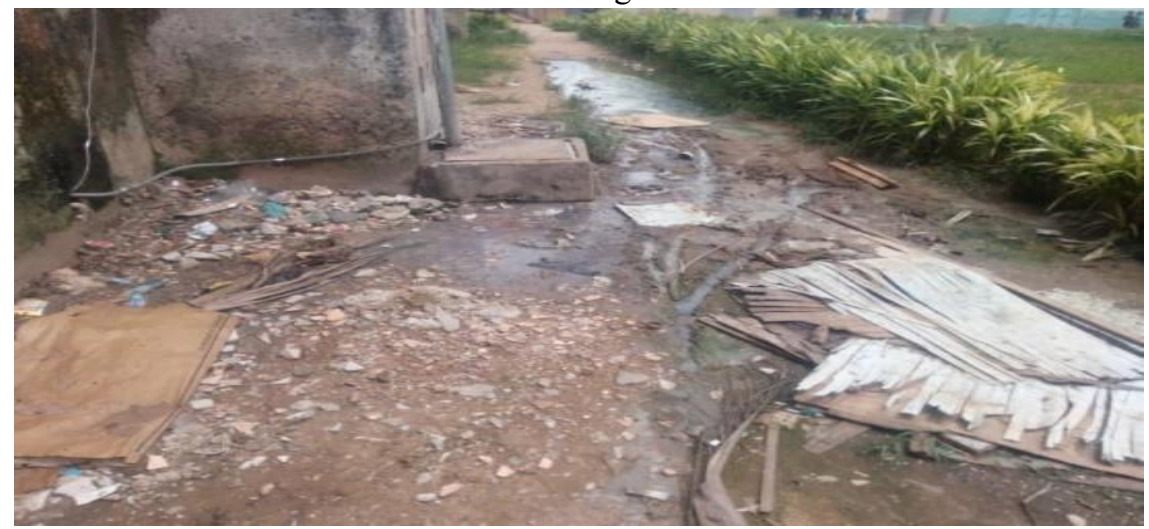

Source : Enquête de l'étude, juillet 2019

\subsection{Le manque de connaissance des syndics}

Le manque de connaissance dans le domaine de la gestion des copropriétés constitue un obstacle chez les syndics dans la mesure où cela crée une ambiguïté dans l'exercice de leur rôle. C'est ce que décrie cet enquêté en 
ces termes : «...C'est lors des élections ou lorsqu'une personne décède qu'on voit le syndic pour nous dire de payer 500 frcs ou 1000 frcs pour soutenir celui qui est décédé sinon je ne vois pas de truc concret même que le syndic a fait. Je ne vois pas le rôle qu'il joue » (Femme, 40 ans, locatrice à Abobo Quatre Etages).

D'après ce discours : "Le syndic fait une gestion interne à telle enseigne que lorsqu'on les poursuit pour faire des réunions, ils refusent. Depuis qu'ils sont élus, on n'a jamais eu de rencontres. Aucun bilan n'est fait ». (Femme, locatrice, résidente au grand bloc d'Adjamé). Au travers de ces dires, c'est la gestion du syndic qui est remise en cause par les résidents.

Pour l'acteur institutionnel : "L'entretien actuellement est laissé pour compte. Les gens font de ça un business. C'est-à-dire un groupe d'individus qui s'entend, qui se disent syndic, se sucrent sur le dos des gens et ne font pas le travail de manière professionnel »(Homme, agent de la SICOGI).

Ces discours laissent transparaitre l'existence de rapport conflictuel entre les catégories sociales interagissant au sein de l'espace. Aussi, ces dires révèlent-ils l'ambigüité des syndics dans l'exercice de leur rôle dans la mesure où l'intérêt porté à la gestion des copropriétés par les membres cache souvent des enjeux d'ordre économique, reléguant donc l'entretien pour lequel ils ont été institués au second plan. Cette attitude de la part des membres des syndics explique également le désintérêt dont font preuve les habitants lorsqu'il est question de prendre part aux réunions et aux cotisations. Dès lors, le nonprofessionnalisme des syndics lié à leur manque de connaissance dans le domaine de la gestion a pour conséquence la dégradation des espaces étudiés. La photo 2 ci-après montre l'état du parking des copropriétés d'Adjamé 220 Logements.

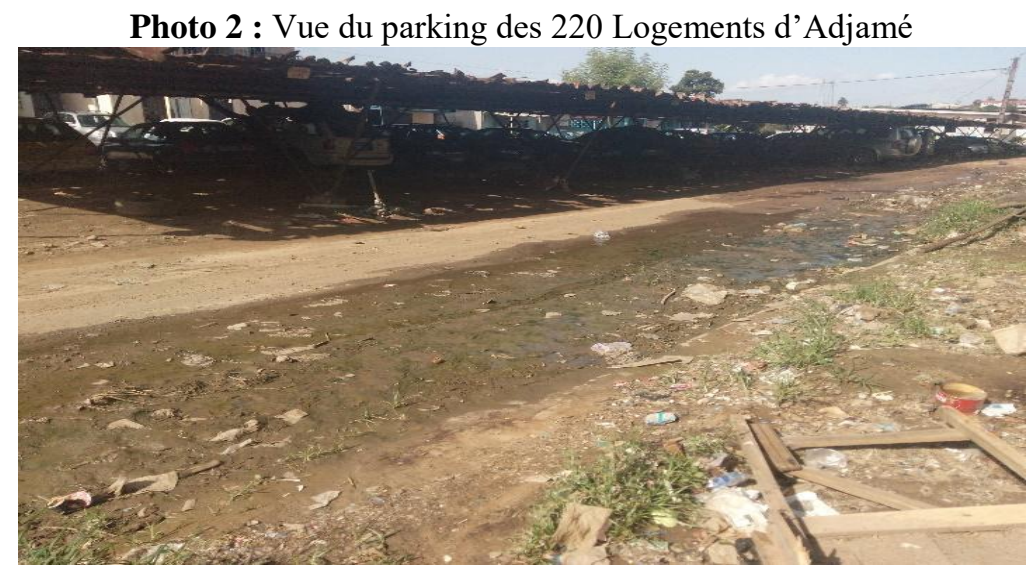

Source : Enquête de l'étude, juillet 2019.

\section{Discussion des Resultats}

Les résultats de cet article se basent sur la théorie de la gouvernance urbaine qui selon Le Gales (1995) est un processus d'acteurs, de groupes 
sociaux et d'institutions en vue d'atteindre des objectifs définis et discutés collectivement. Cette théorie sous-tend un rapport de collaboration entre les différentes parties mobilisées dans la gestion de la chose collective. En effet, la gestion des copropriétés fait partie de la politique de la ville encourageant une démocratie participative, s'appuyant sur les potentialités des habitants (Avenel, 2007). Cette gestion s'inscrivant dans un cadre normatif, établi de façon consensuelle. Cependant dans cette étude, les acteurs chargés de la gestion de l'espace entretiennent des rapports conflictuels. Ces rapports entre acteurs combinés aux stratégies d'entretien mises en place par les syndics ont pour conséquence la dégradation de la chose collective qu'est l'espace résidentiel.

\subsection{Les stratégies d'entretien}

Cet article démontre que les stratégies d'entretien mises en place par les syndics et associations sous-tendent certaines pratiques étant à l'origine de la dégradation des copropriétés SOGEFIHA d'Abobo et SICOGI d'Adjamé 220 Logements.

En accord avec ce résultat, Krepka et al. (2016) mettent en cause les nouvelles formes de gestions comme facteurs de dégradation des copropriétés de la Petite et Grande Ourse de Cocody depuis le retrait de SICOGI. Les auteurs révèlent le décalage entre la gestion assurée par la SICOGI en son temps et celle faite par les copropriétaires depuis sa mise en liquidation. Ils dénoncent l'usage du bâtiment comme emplacement publicitaire et support des dispositifs privés, la mise en location privative du local destiné au recouvrement des loyers par la SICOGI, la transformation du séchoir en magasins commerciaux et pièce locative, l'occupation du parvis et des couloirs des bâtiments à des fins d'activités économiques, la transformation de la buanderie en un lieu de résidence, la transformation du bureau des copropriétaires en magasin et les locations et sous-locations des appartements.

Toutefois, Kouamé (1983) révèle le rapport des habitants à l'espace en s'appuyant sur leurs pratiques. Il s'agit des modifications effectuées à l'intérieur du logement à cause de la rotation importante d'individus qui y vivent et de la persistance d'anciennes pratiques au niveau des parties communes issues de leur trajectoire résidentielle.

Par ailleurs, Bassa (2015) trouve que le véritable problème est que la gestion des copropriétés est confiée aux résidents qui ne s'occupent que de l'intérieur de leurs logements tandis que l'extérieur des bâtiments laisse une image désolante. Pour eux, seul l'entretien de l'espace privatif leur incombe car leur propriété ne se limite qu'à l'intérieur du logement. Les espaces communs étant la propriété collective, nul ne se sent directement responsable de leur entretien. 


\subsection{Le manque de connaissance des syndics}

Comme démontré dans cet article, la dégradation est la résultante du manque de connaissance des syndics. Hsieh (2009) est en accord avec cette thèse lorsqu'il dénonce le désengagement de l'Etat de l'entretien comme facteur de délabrement des copropriétés. Selon l'auteur, le fait que les copropriétaires soient livrés à eux-mêmes dans la gestion de leur cadre de vie alors qu'ils n'ont pas de connaissance dans ce domaine constitue la cause du phénomène. Chen (2011), abondant dans le même sens, soutient que l'implication de l'Etat est un élément important au bon fonctionnement des copropriétés. Sur la base du rapport de collaboration entre les acteurs dans le cadre de la gouvernance, une collaboration interstructurelle s'impose pour la bonne gestion des copropriétés. Cette collaboration passe par la formation des copropriétaires et/ou des résidents sur l'organisation des copropriétés (Major, 1992 ; Kennedy, 1995 ; Lai et Chan 2004 cités par Simon 2015).

\section{Conclusion}

Au terme de cet article, il ressort que la dégradation est un produit social résultant des modes de gestion des copropriétés SOGEFIHA d'Abobo et SICOGI d'Adjamé et du manque de connaissance des syndics chargés de cette gestion. L'approche qualitative adoptée a permis de mettre en exergue les stratégies mises en place par les syndics et les associations dans la gestion de ces espaces. A l'analyse, le rapport des résidents à l'espace montre l'existence de conflits entre ces derniers et les syndics dont la résultante est la dégradation. Les moyens de pallier la dégradation ne sont pas abordés dans ce travail. La prise en compte de cet aspect pourrait constituer une perspective de recherche pour les études ultérieures.

\section{References:}

1. AVENEL, C. (2007). « La politique de la ville et ses destinataires : une politique de gestion d'un sentiment d'abandon», Informations sociales. 5, (141), pp. 136-147. Consulté le 04 Avril 2019. URL : https://www.cairn.info/revues-informations-sociales-2007-5-pages136.htm.

2. BARDIN, L. (2007). L'analyse de contenu. Paris : PUF, Collection Quadrillage.

3. BASSA, A. (2015). Cité des Arts de Cocody : du prestige au délabrement. Consulté le 07 Avril 2019. URL : https://www.Akody.com/culture/news/cite-des-arts-de-cocody-duprestige-au-delabrement-267668.

4. CHEN, S. Y. (2011). Common interest development and the changing roles of government and market in planning. Urban Studies, 48 (16), pp. 3599-3612. 
5. HAERINGER, P. (1985). «Vingt-cinq ans de politique urbaine à Abidjan ou la tentation de l'urbanisme intégral ». Politique Africaine, (17), pp 20-40.

6. HSIEH, H. R. (2009). «Problèmes et améliorations proposées concernant la gestion des copropriétés à Taiwan ». Habitat International, 33(1), pp. 73-80.

7. KOUAME, N'G. (1983). «Devant et derrière les murs(I) : la qualité de la vie dans l'habitat économique d'Abidjan. Abidjan au coin de la rue ». Cahiers de l'ORSTOM, 19(4), pp. 449-458.

8. KREPKA, D. J. Z, TAGRO, M-J. \& KOUAME, A. (2016). « Formes et enjeux de gestion des espaces en copropriété de Cocody Petite et Grande Hourse ». RSS-PASRES, pp. 110-123.

9. LE GALES, P. (1995). «Du gouvernement des villes à la gouvernance urbaine $»$. Revue française de science politique, 45(1), pp. 57-95.

10. MANOU-SAVINA, A. (1989). «Éléments pour une histoire de la cour commune en milieu urbain ». Tropiques, lieux et liens, ORSTOM, Paris, pp. 310-317.

11. N'DA, P. (2015). Recherche et méthodologie en sciences sociales et humaines : Réussir sa thèse, son mémoire de master ou professionnel, et son article. Paris : L'Harmattan.

12. PARENTAU, R. \& CHARBONNEAU, F. (1992). «Abidjan : une politique de l'habitat au service du plan urbain ». Cahiers de Géographie du Quebec,36(99), pp.415-437. http://dx.doi.org/10.7202/022293ar.

13. PARSONS, T. (1951). The Social System. Glencoe : Free Press.

14. PREVOST, M. (1985). "Politique de l'habitat: d'un âge à un autre». Cités africaines, $\mathrm{n}^{\circ} 2$, janvier-mars, pp. 24-28.

15. SIMON, E. (2013). "Quelques enjeux autours des copropriétés et de leur dégradation », Université de Lorraine, Nancy, France, Consulté le 04 avril 2019. URL : https://halshs.archives-ouvertes.fr/halshs01098315.

16. SIMON, E. (2015). "Dégradation des copropriétés et politiques publiques dédiées : revue de littérature anglophone ». Consulté le 04 Avril 2019. URL : https://halshs.archives-ouvertes.fr/hal-01199785.

17. SOUMAHORO, C. (1997). "Modèle et types d'habitat à loyers modérés à Abidjan : l'Etat propose, les habitants et les constructeurs disposent... In : CONTAMIN BERNARD (ED.), Memel-Fotê H. (ed.) Le modèle ivoirien en question : crises, ajustements, recompositions. Paris : Karthala, Osrtom, pp. 515-528, (Hommes et sociétés), ISBN 286537-748-2. 